\title{
The effect of object size on the sensitivity of single photon emission computed tomography: comparison of two CZT cardiac cameras and an Anger scintillation camera
}

Elazar A Bienenstock ${ }^{1,2^{*}}$ and Marguerite Ennis ${ }^{3}$

* Correspondence:

dr.bienenstock@gmail.com

'Department of Nuclear Medicine, Etobicoke General Hospital of William Osler Health System, 101 Humber College Blvd., Markham, ON, Canada M9V 1 R8

${ }^{2}$ Scarborough Cardiac Diagnostic Centre, 2391 Eglinton Ave E, Toronto, ON, Canada M1K 2M5 Full list of author information is available at the end of the article

\begin{abstract}
Background: Heart sizes vary greatly across the spectrum of patients referred for myocardial perfusion imaging. We therefore performed a phantom study to explore under controlled circumstances how count rates change when different volumes containing the same amount of activity are scanned. Two dedicated cadmium-zinc-telluride cameras, the D-SPECT (Spectrum Dynamics, Caesarea, Israel) and Discovery 530c (D530c, GE Healthcare, Haifa, Israel), and the conventional SPECT Anger (A-SPECT, GE Healthcare, Haifa, Israel) camera are included in the study.
\end{abstract}

Methods: Different heart sizes were represented by syringes of various column heights mimicking a range of cardiac diameters. Syringes with fixed activity were scanned at five different volumes by successively adding non-radioactive water to the syringes. This procedure was repeated five times on each of the three cameras. Raw count rates were recorded for each scan to determine whether count rates changed with syringe column height.

Results: Using mixed-effect regression modeling, a linear relationship was found between count rate and water column height. For the D-SPECT, D530c, and A-SPECT, the changes in count rate for each centimeter increase in water column height were $-1.75,+0.28$, and -0.022 kilocounts per min per MBq, respectively ( $95 \%$ confidence intervals -1.89 to $-1.61,0.19$ to 0.36 , and -0.035 to -0.009 ); all effects are significantly different from each other and significantly different from zero. Average coefficients of variation were $0.080,0.028$, and 0.009 .

Conclusions: The D-SPECT demonstrated a significant progressive increase in count rate related to decreasing size of the imaged object. D530c count rate increased slightly with increasing column height. The Anger SPECT showed minimally increased count rates with decreasing column height, an order of magnitude smaller than the D-SPECT based on their relative coefficients of variation.

Keywords: CZT camera; Coronary artery disease; Myocardial perfusion imaging; High-speed SPECT; Heart size

\section{空

2014 Bienenstock and Ennis; licensee Springer. This is an Open Access article distributed under the terms of the Creative Commons Attribution License (http://creativecommons.org/licenses/by/4.0), which permits unrestricted use, distribution, and reproduction in any medium, provided the original work is properly credited. 


\section{Background}

Coronary artery disease is a leading cause of morbidity and mortality, particularly in advanced countries [1,2] Myocardial perfusion scans are a key tool for the diagnosis and risk assessment of coronary artery disease. The expansion of imaging technology and interest in early disease detection have led to an estimated 9.1 million tests being performed annually in the United States in 2008 [3,4]. The Anger scintillation gammacamera design has been utilized since 1957 [5]. Recently two direct-conversion cadmiumzinc-telluride (CZT) detector cameras have become available: the D-SPECT (Spectrum Dynamics, Caesarea Israel) and Discovery 530c (D530c, GE Healthcare, Haifa, Israel). These have several advantages over scintillation gamma-cameras, including much faster scans [6].

Heart sizes vary greatly across the spectrum of patients referred for myocardial perfusion imaging. One standard deviation for myocardial mass in normal adult males ranges from 92 to $190 \mathrm{~g}$ [7]. The population undergoing myocardial perfusion scans includes normal women, which extends this range downward. The scanned population also includes a significant number of obese, diabetic, hypertensive, and ischemic patients at risk for left ventricular (LV) dilation, which extends the range upwards.

The development and enthusiastic acceptance of ultrasensitive cardiac CZT cameras highlights the importance of scan speed in contemporary nuclear medicine practice. Higher scan speed increases patient comfort, convenience, and throughput; reduces motion artifact; or facilitates radiotracer dose reduction. Recently, the scan speed of these CZT cameras was compared [8].

It has been our experience that when using the automated myocardial count level imaging function of the D-SPECT, stress scans are occasionally completed in less than 1 min rather than the average 2 min when using rest and stress doses of approximately 333 and $999 \mathrm{MBq}$, respectively. Paradoxically, these short duration scans demonstrated images of excellent quality. The fact that these short acquisition times tended to occur in small patients raised the question of a possible relationship between cardiac size and myocardial count rate. To investigate this, we performed a phantom study using a fixed amount of activity spread over increasing volumes to determine the effect of object size alone on count rate. We included the D-SPECT, D530c, and the conventional Anger SPECT (A-SPECT) camera in the study because, while it is known that these three cameras have different sensitivities [8], their response to changes in object size has not been compared. Based on a review of the camera mechanisms, we hypothesize that only for the D-SPECT, count rate would increase significantly with decreasing heart size.

\section{Methods}

Instrumentation

This study involved the use of a the D-SPECT camera at the Scarborough Cardiac Diagnostic Center (SCDC) and a D530c camera at Etobicoke General Hospital (EGH). The Anger SPECT (A-SPECT) camera used in this study was a Millennium VG (GE Healthcare, Haifa, Israel) at EGH. All were in Toronto, ON, Canada. The dose calibrator from SCDC was transported to the EGH to calibrate the dose calibrators to each other by measuring various activities in both devices in rapid succession. The D-SPECT and D530c both use identical square CZT modules. Each module consists of a 1616 pixelated CZT 
crystal backed by a proprietary application-specific integrated circuit. The D-SPECT employs nine rotating detector columns (DCs) in an L-shaped array that translates between rotations to view spaces between detectors. Each rectangular DC consists of four vertically stacked CZT modules behind a very-high-sensitivity parallel-hole tungsten collimator. Each square collimator hole corresponds to one pixel etched into the CZT crystal surface. The D-SPECT requires a brief pre-scan following which the technologist draws a region of interest (ROI) to define the LV for cardio-centric DC movements [9]. Utilizing countrate information from the pre-scan, the D-SPECT adjusts scan time in 1-s increments to attain a predetermined number of counts. The D530c employs an L-shaped array of 19 stationary pinhole units arranged in three rows. Each unit consists of a tungsten-tipped lead-bodied pinhole collimator with an effective aperture of $5.1 \mathrm{~mm}$, projecting onto four CZT modules arranged in a 22 square [10].

\section{Preparation of syringe sets and data acquisition}

The ready and reproducible production of different sized objects with intrinsically equal activity was achieved by repeatedly adding non-radioactive water to successively lengthen the activity column of a syringe containing Tc-99 $\mathrm{m}$ as follows. Two syringes were attached to a three-way stopcock at right angles paralleling both arms of the L-shaped CZT detectors and the Anger camera detectors in L mode at the start position. The setup at the EGH included 10-mL (B to D) syringes measuring $82 \mathrm{~mm}$ from the stopcock hinge to the 10-mL mark. At SCDC, 12-mL (Terumo, Shibuya, Japan) syringes were used measuring $78 \mathrm{~mm}$ from the stopcock hinge to the $10-\mathrm{mL}$ mark (Figure 1). A solution of 14 to $46 \mathrm{MBq}$ of Tc-99m in less than $1 \mathrm{cc}$ of water was prepared and approximately half was injected into each of the two empty syringes. Water was added to both syringes through the 3rd stopcock port, topping-up both syringes to $1 \mathrm{cc}$. The horizontal syringe was taped to the imaging table with the plunger head pointing towards the vertical detector arm. The vertical syringe s plunger required pruning to avoid collision with the upper CZT detector arms as the syringe is filled with water. Following the scan, $2 \mathrm{cc}$ of water was added to each syringe. The time of dose calibration and start of each scan were recorded for decay correction. Without moving the syringe set, this process was repeated until five unique paired-column-height measurements of the same activity were obtained for each syringe set. Five syringe sets were scanned on each of the three cameras for a total of 75 measurements $(5$ column heights 5 syringe-sets 3 cameras). The column height measurements were 3.4 to $7.5 \mathrm{~cm}$ for the D-SPECT and 3.0 to $7.8 \mathrm{~cm}$ for the D530c and A-SPECT, due to the fact that the dimensions of the syringes and stopcocks supplied at SCDC were slightly different from those at the EGH. The arbitrary syringe set activities overlap the clinically encountered myocardial activity range of 8 to $24 \mathrm{MBq}$, based on $0.6 \%$ to $1.8 \%$ myocardial uptake [11] of 1,332 MBq Tetrofosmin-Tc-99m. Visual inspection of the images confirmed homogeneous activity throughout the extended water columns after each infusion of non-radioactive water, indicating good mixing during water injection. The entire experiment was conducted in the absence of external attenuation, eliminating potential confounding effects of variable external attenuation.

Scan duration was $2 \mathrm{~min}$ on both CZT cameras and $10 \mathrm{~min}$ and $13 \mathrm{~s}$ on the A-SPECT. A 2-min duration matches average D-SPECT clinical stress scan time and has been suggested for the D530c [6]. Ten minutes is at the lower limit of conventional A-SPECT 


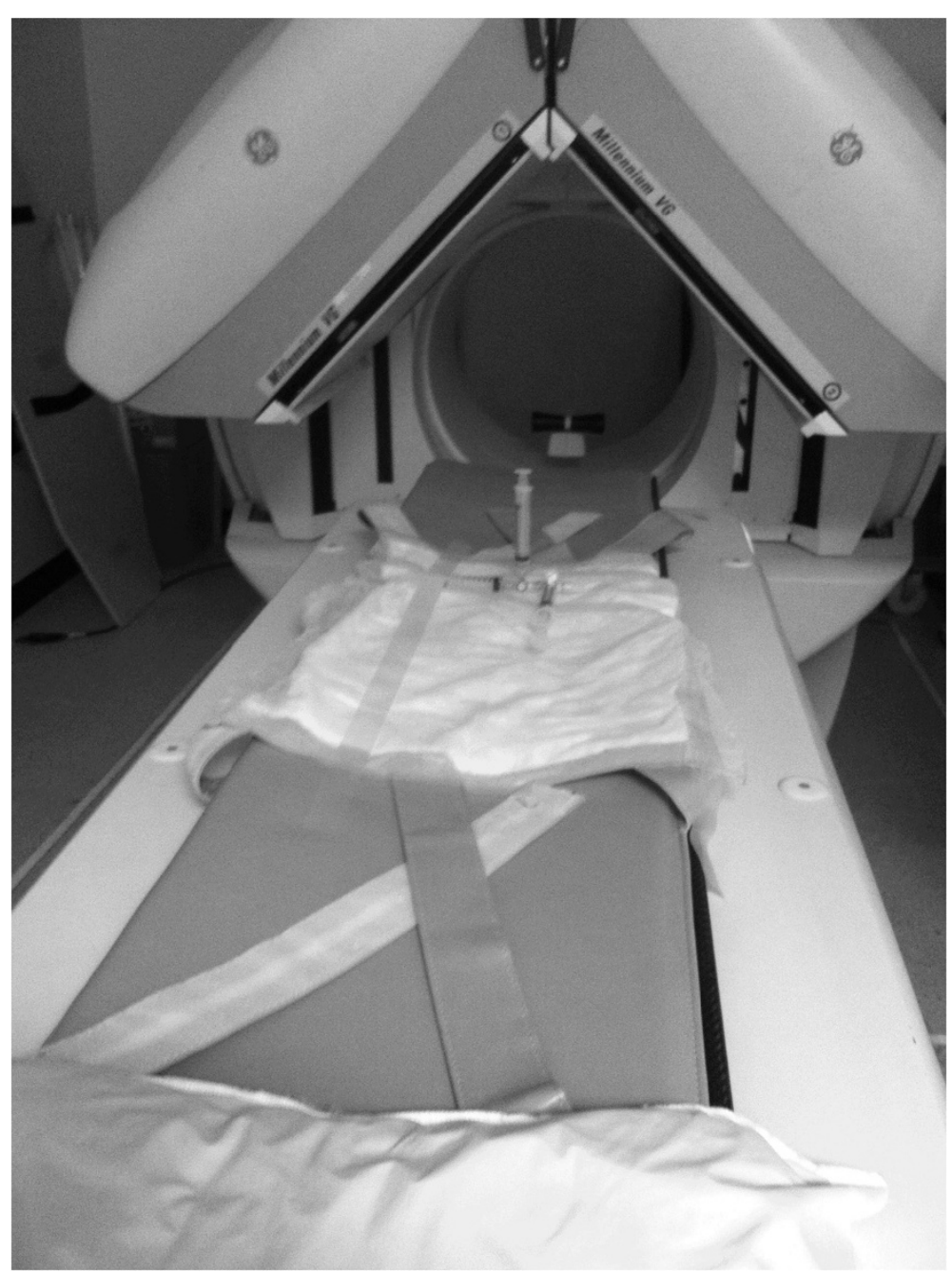

Figure 1 Experimental setup. Syringe-set at $1 \mathrm{cc}$ in the A-SPECT camera just before rotating the detector to the start position.

stress scan duration. Shorter scan times would underestimate A-SPECT and D-SPECT count rates by increasing the proportion of lag time due to detector motion. The D530c s fixed detectors suffer no lag time.

\section{D-SPECT syringe imaging}

Superimposed on the D-SPECT's pre-scan is a vendor-rendered circle centered $5.8 \mathrm{~cm}$ from the anterior camera surface and $5.2 \mathrm{~cm}$ from the lateral surface, indicating preferred heart position. For each series of measurements, the syringe set was positioned with the stopcock hinge at the center of this circle and not moved until the series was completed. On each pre-scan a tight ROI was drawn around the activity columns of the syringe set in a standard manner to avoid variability due to the shape of the ROI.

\section{D530c syringe imaging}

Optimal heart position is indicated by vendor-rendered crosshairs at 12 and $15 \mathrm{~cm}$ from the anterior and lateral camera surfaces, respectively, in plane with the central 
row of pinholes. For each series of measurements, the syringe set was positioned such that the stopcock hinge was at the crosshairs and it was not moved until the series was completed.

\section{A-SPECT syringe imaging}

A central point was chosen on the imaging table, and the syringe set is positioned at this point for each series. The syringe set was not moved between scans of the same series. Scans were performed with 30 stops (60 projections) for $18 \mathrm{~s}$. Rotation diameter was $18 \mathrm{~cm}$.

\section{Count quantification}

Raw projection counts purely summed from all detectors were compared. Because all activity was from the syringe-sets, reconstruction to exclude background activity was unnecessary. As the D-SPECT scan progresses, the growing count number is refreshed on the acquisition monitor. The final value after scan completion was used. GE software was used to sum counts from the D530cs 19 pinholes and from A-SPECTs 60 projections.

The data for each camera consisted of 5 series, each series consisting of the count rates corresponding to 5 different water column heights. The measured counts were normalized to an activity of $1 \mathrm{MBq}$ by using the dose calibrator activity measurement for each series, decay-corrected to the time of the scans. Counts were further divided by the scan duration to obtain counts per minute per megabecquerel.

\section{Statistical analysis}

The coefficient of variation $(\mathrm{CV})$ of the count rates was calculated for each series and the hypotheses that the CV's were the same for all three cameras tested using ANOVA. Regression modeling was used to obtain estimates of the count rate vs. object size relationship, in the form of a mixed model that accounted for the fact that observations were not completely independent but are correlated within the series. We fitted different intercepts and slopes for the three cameras and used random intercept effects for the series. Variances were allowed to differ by camera. The model was fitted by maximum likelihood and parameterized in two ways: one to test whether the slopes of the three cameras were significantly different from each other (using interactions) and the other to test whether the slopes differed significantly from zero. Residual analyses were satisfactory. All analyses were performed using S-PLUS 6.2 (Insightful Corp, Seattle, USA).

\section{Results}

The relationship between count rates and the water column height is shown in Figure 2 with data in Table 1. The estimated slopes for the D-SPECT, D530c, and A-SPECT respectively from the mixed-effects model were $-1.75,0.28$, and -0.022 kilocounts per min per $\mathrm{MBq}$ for each centimeter increase in water column height (95\% confidence intervals -1.89 to $-1.61,0.19$ to 0.36 , and -0.035 to -0.009 , respectively); these slopes were all significantly different from each other (data not shown) and significantly different from zero (Table 2). Thus, D-SPECT and A-SPECT's count rates increased as the water volume decreased while D530c showed the opposite pattern. The average CV's were 

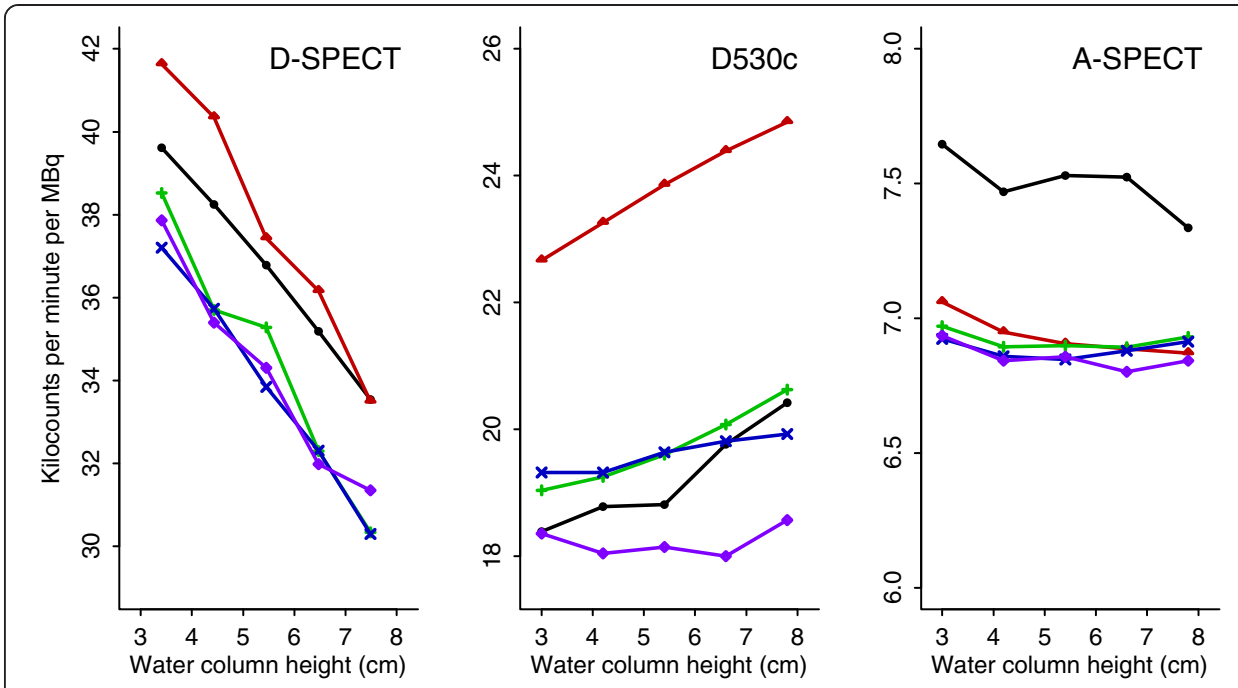

Figure 2 Count rates as a function of syringe water column height. A series of measurements at five different syringe heights was obtained by successively adding non-radioactive water to the syringe to lengthen the activity column. Five series are shown for each of the three cameras D-SPECT, D530C, and A-SPECT.

Table 1 Count rates corresponding to different syringe water column heights

\begin{tabular}{|c|c|c|c|c|c|c|c|c|}
\hline \multirow[t]{7}{*}{ D-SPECT } & Water column height & $3.4 \mathrm{~cm}$ & $4.4 \mathrm{~cm}$ & $5.5 \mathrm{~cm}$ & $6.5 \mathrm{~cm}$ & $7.5 \mathrm{~cm}$ & & \\
\hline & & \multicolumn{5}{|c|}{ Kilocounts per minute per megabecquerel } & $\mathrm{CV}^{\mathrm{a}}$ & Mean $\mathrm{CV}^{\mathrm{b}}$ \\
\hline & Series 1 & 39.62 & 38.25 & 36.79 & 35.19 & 33.54 & 0.066 & 0.080 \\
\hline & Series 2 & 41.63 & 40.35 & 37.43 & 36.16 & 33.49 & 0.086 & \\
\hline & Series 3 & 38.53 & 35.70 & 35.28 & 32.30 & 30.33 & 0.092 & \\
\hline & Series 4 & 37.20 & 35.73 & 33.84 & 32.31 & 30.30 & 0.081 & \\
\hline & Series 5 & 37.86 & 35.39 & 34.30 & 31.98 & 31.35 & 0.077 & \\
\hline \multirow[t]{7}{*}{ D530c } & Water column height & $3 \mathrm{~cm}$ & $4.2 \mathrm{~cm}$ & $5.4 \mathrm{~cm}$ & $6.6 \mathrm{~cm}$ & $7.8 \mathrm{~cm}$ & & \\
\hline & & \multicolumn{5}{|c|}{ Kilocounts per minute per megabecquerel } & $\mathrm{CV}^{\mathrm{a}}$ & Mean $C V^{b}$ \\
\hline & Series 1 & 18.38 & 18.78 & 18.81 & 19.76 & 20.42 & 0.043 & 0.028 \\
\hline & Series 2 & 22.66 & 23.26 & 23.86 & 24.39 & 24.84 & 0.037 & \\
\hline & Series 3 & 19.03 & 19.25 & 19.60 & 20.07 & 20.62 & 0.033 & \\
\hline & Series 4 & 19.32 & 19.32 & 19.63 & 19.81 & 19.92 & 0.014 & \\
\hline & Series 5 & 18.35 & 18.04 & 18.14 & 18.00 & 18.57 & 0.013 & \\
\hline \multirow[t]{7}{*}{ A-SPECT } & Water column height & $3 \mathrm{~cm}$ & $4.2 \mathrm{~cm}$ & $5.4 \mathrm{~cm}$ & $6.6 \mathrm{~cm}$ & $7.8 \mathrm{~cm}$ & & \\
\hline & & \multicolumn{5}{|c|}{ Kilocounts per minute per megabecquerel } & $\mathrm{CV}^{\mathrm{a}}$ & Mean $\mathrm{CV}^{\mathrm{b}}$ \\
\hline & Series 1 & 7.65 & 7.47 & 7.53 & 7.52 & 7.33 & 0.015 & 0.009 \\
\hline & Series 2 & 7.06 & 6.95 & 6.91 & 6.89 & 6.87 & 0.011 & \\
\hline & Series 3 & 6.97 & 6.89 & 6.90 & 6.89 & 6.93 & 0.005 & \\
\hline & Series 4 & 6.92 & 6.86 & 6.85 & 6.88 & 6.91 & 0.005 & \\
\hline & Series 5 & 6.94 & 6.84 & 6.86 & 6.80 & 6.84 & 0.007 & \\
\hline
\end{tabular}

${ }^{a} \mathrm{CV}$, coefficient of variation.

${ }^{\mathrm{b}} P$-value $<0.0001$ for ANOVA test that the mean $\mathrm{CV}$ of the three cameras are all equal. 
Table 2 Modeled relationship between count rate and water column height

\begin{tabular}{|c|c|c|c|c|}
\hline Fixed effects & Coefficient value & Standard error & Degrees of freedom & $P$-value \\
\hline Intercept & 44.917 & 0.776 & 57 & $<.0001$ \\
\hline D530c vs. D-SPECT & -26.300 & 1.049 & 12 & $<.0001$ \\
\hline A-SPECT vs. D-SPECT & -37.779 & 1.021 & 12 & $<.0001$ \\
\hline Slope for the D-SPECT & -1.747 & 0.072 & 57 & $<.0001$ \\
\hline Slope for the D530c & 0.277 & 0.043 & 57 & $<.0001$ \\
\hline Slope for the A-SPECT & -0.022 & 0.007 & 57 & 0.0016 \\
\hline Random effects & Standard deviation & \multicolumn{3}{|c|}{ Standard deviation adjustment per camera } \\
\hline Intercept & 1.42 & D-SPECT & D530c & A-SPECT \\
\hline Residual & 0.497 & 1 & 0.706 & 0.108 \\
\hline
\end{tabular}

$0.080,0.028$, and 0.009 , respectively $(P<0.0001$ for the null hypothesis that the CV for the three cameras were equal, Table 1 ). The CV's show that, while both D-SPECT and A-SPECT had negative slopes, the D-SPECT slope was much steeper relative to the average sensitivity of the camera than the A-SPECT slope (average sensitivities for the D-SPECT, D530c, and A-SPECT were 35.4, 20.1, and 7.02 kilocounts per min per $\mathrm{MBq}$, respectively).

\section{Discussion}

The D-SPECT demonstrated significantly improved count rates with decreasing activity volume, a feature not previously encountered in nuclear medicine cameras. This camera feature is explained by the D-SPECTs internal mechanics. According to Spectrum Dynamics (personal communication), each of the D-SPECT's nine DCs initially acquires images at 120 steps across a 110 arc, with equal time spent at each position. After the pre-scan, the computer adjusts DC movement so that $60 \%$ to $70 \%$ of angles view the LV as defined by the technologist-rendered ROI and 30\% to 40\% view the remainder of the chest. We can describe the $60 \%$ to $70 \%$ left ventricular views as the central portion of the field of view (CPFOV). As the heart size decreases, the DC angular intervals in the CPFOV become finer and more closely stacked causing increased overlap of consecutive DC images, at the expense of slightly coarser peripheral sampling. Thus, the virtual detector contracts or expands the CPFOV in response to heart size. Denser oversampling of smaller hearts increases cardiac count rate. The increase in sampling density and count rate with CPFOV shrinkage resembles thickening of the detector as it contracts, like a muscle or rubber band. Another analogy that may capture this effect is a searchlight gliding over a field. A smaller search area enjoys improved illumination because the searchlight dwells longer at each location.

In contrast to the D-SPECT, fixed field of view cameras (D530c and A-SPECT) did not demonstrate similarly improved count rates with decreased column height. In fact, D530c count-rate increased slightly with increased column height. Further study may help assess whether this occurred because more of the activity was perpendicular to the pinholes with increased column height. The A-SPECT's count rates showed a small but statistically significant increase with decreasing column height. The increase is unlikely 
to be important clinically and is likely due to decreased self-attenuation by the smaller column. Although decreased self-attenuation may also increase D-SPECT count rates, the small scale of this effect cannot account for the much larger increase in count rate observed for the D-SPECT with decreased column height. It should be noted that, because these cameras have different resolution and efficiency characteristics, count rate does not reflect the resulting contrast to noise ratios that can be achieved for a given acquisition time.

Beyond the D-SPECT's adaptable virtual detector, there are supplemental causes for the rapid and high quality scans of small hearts that originally lead to this investigation. Images of smaller hearts are less noisy due to a higher count density, since total LV counts are shared by fewer milliliters or voxels. Additionally, small hearts are associated with smaller patients [12]. Decreased soft tissue attenuation in small patients allows more photons to escape the body and reduces scattered photons that deliver incorrectly assigned points of origin. This leads to higher count rates and better resolution. Voxel count density and attenuation affect all camera systems equally and unrelated to the D-SPECT s increased count rate in small hearts identified in our phantom study.

CZT cameras possess small fields of view that are quite sensitive to heart position [13]. Smaller patients' hearts are more ideally positioned in the field of view. Additionally, closer proximity to the detector should improve image resolution and count rate for both cameras. Because only CZT cameras scan in direct chest contact, the improved heart-detector proximity due to decreased chest size in smaller patients is proportionately much more significant for CZT than A-SPECT cameras. For the D-SPECT, the center of the circle likely allows all nine DCs to view the heart from the closest average distance. Although D-SPECT uses a parallel hole collimator and Anger camera parallel hole collimator efficiency does not change with distance, D-SPECT sensitivity should decrease with increased distance from the heart. This is because of the size difference between A-SPECT and D-SPECT collimators. The Anger cameras have large detectors where the sensitivity lost per individual collimator-hole is offset by an equal increase in the number of collimator holes viewing an object as it recedes. Since the D-SPECT's 4-cm wide DCs do not span the average internal end systolic LV diameter [14], the number of collimator holes exposed to the heart does not increase in step with the decrease in individual collimator hole sensitivity as the object recedes. For the D530c, the crosshairs location presumably minimizes average myocardial displacement from the 19 pinhole apertures, since pinhole sensitivity falls with increasing angle and distance [15].

From a resource management perspective, extra high quality scans are overexposed and present an opportunity to decrease radiopharmaceutical dose and/or scan-time. Although all cameras register higher count rates in smaller patients, only the D-SPECT capitalizes on this by shortening scan duration, thanks to its scan strategy that targets LV counts rather than a predetermined exposure time. The D-SPECT scan times could potentially be shortened even further for smaller hearts by targeting count density rather than total counts. LV volume might be estimated from the pre-scan. BMI input could further refine count-targets because improved heart detector proximity and less scatter in smaller patients could allow scan completion with lower than normal countdensity. Currently, the Spectrum Dynamics recommends outlining the pre-scan LV with generous margins. As this study indicates, a tighter LV margin will increase count-rate by concentrating the CPFOV. Further study may reveal if tighter ROIs can be drawn to increase scan speed without degrading image quality. 
In locales where heart sizes are smaller due to ethnic variations [16], this may mitigate slower scan speed associated with D-SPECT versions employing fewer DCs.

Other cameras could potentially personalize scan time without adding a timeconsuming pre-scan by measuring LV count rate during early acquisition, provided that the exposure time adjustments described above could be completed well before the scan would normally end. D530c is particularly well suited for this because its inherent full-time SPECT configuration permits scan termination at any instant.

\section{Limitations}

While different sized cardiac phantoms would be ideal, they are not available. Initially we attempted to use different sized hollow spheres to approximate the size and shape of the left ventricle. However, small random variations in dose-calibrator sphere-activity assays marred count-rate/megabecquerel comparisons between the different sized spheres. Since the syringe set only required repositioning five times for each camera, and care was taken to place it at the same spot each time, variability due to source location was minimal. Location was undefined only for the A-SPECT, but is unlikely to be relevant. The D-SPECT ROI was allowed to vary with object size as it would in practice, but its shape was standardized to avoid differences in ROI between repeat scans.

\section{Conclusions}

This study confirms a significant volume effect on count rate for the D-SPECT due to its mechanically adaptable virtual detector. This design feature provides increased scope for acquisition optimization and has not been previously encountered in nuclear medicine cameras.

Smaller hearts increase the D-SPECT's count rates due to its mechanics and higher count-rates increase the D-SPECT's scan speed due to its scan strategy. Therefore, for any scan-speed evaluation or comparison of the D-SPECT with other cameras, heartsize or object size must be taken into account.

Targeting count density rather than absolute LV counts may optimize D-SPECT scan speed for all patient sizes. BMI input may further reduce count targets and scan time by leveraging multifactorial improved spatial resolution in small patients. The D530c is well suited to switch from a time-based to a count-based scan strategy to also personalize scan times. Benefits of personalizing scan time include shorter average scan time and extended scan time when needed.

\section{Abbreviations}

A-SPECT: Anger SPECT; BMI: body mass index; CPFOV: central portion of the field of view; CV: coefficient of variation; CZT: cadmium-zinc-telluride; D530c: Discovery 530c; DC: detector column; EGH: Etobicoke General Hospital; LV: left ventricular; ROI: region of interest; SCDC: Scarborough Cardiac Diagnostic Center.

Competing interests

The authors declare that they have no competing interests.

Authors' contributions

EB conceived the study, performed the experiments and drafted the manuscript. ME participated in the design of the

study, performed the statistical analysis, and revised the manuscript. Both authors read and approved the final manuscript.

\section{Acknowledgements}

Thanks to Dr. Curtis Caldwell and Dr. Mathews Fish for their helpful input. Thanks to Lori Lenon and Mansour Damani for the assistance with preparation and scanning of spheres and syringes at the Scarborough Cardiac Diagnostic Center and to Anita Dillon, Hugh Lee and Cain Szeto for the same at the EGH. Thanks to Dr. Jonathan Sachs and Reuven Brener of GE Healthcare and to Nathaniel Roth and Adi Nachmani of Spectrum Dynamics, for providing useful information and feedback. 
Author details

'Department of Nuclear Medicine, Etobicoke General Hospital of William Osler Health System, 101 Humber College Blvd., Markham, ON, Canada M9V 1R8. ${ }^{2}$ Scarborough Cardiac Diagnostic Centre, 2391 Eglinton Ave E, Toronto, ON, Canada M1K 2M5. ㅅApplied Statistician, 9227 Kennedy Rd, Markham, ON, Canada L3R 6H9.

Received: 20 June 2014 Accepted: 10 October 2014

Published online: 31 December 2014

\section{References}

1. Rosamond W, Flegal K, Furie K, Go A, Greenlund K, Haase N, Hailpern SM, Ho M, Howard V, Kissela B, Kittner S, Lloyd-Jones D, McDermott M, Meigs J, Moy C, Nichol G, O Donnell C, Roger V, Sorlie P, Steinberger J, Thom T, Wilson M, Hong Y: Heart disease and stroke statistics-2008 update: a report from the American Heart Association Statistics Committee and Stroke Statistics Subcommittee. Circulation 2008, 117:e25 e146.

2. Lloyd-Jones D, Adams RJ, Brown TM,Carnethon M, Dai S, De Simone G, Ferguson TB, Ford E, Furie K, Gillespie C, Go A, Greenlund K, Haase N, Hailpern S, Ho PM, Howard V, Kissela B, Kittner S, Lackland D, Lisabeth L, Marelli A, McDermott MM, Meigs J, Mozaffarian D, Mussolino M, Nichol G, Roger VL, Rosamond W, Sacco R, Sorlie P, et al: Heart disease and stroke statistics-2010 update: a report from the American Heart Association. Circulation 2010, 121:e46 e215.

3. de Gonzalez AM, Kim KP, Smith-Bindman R, McAreavey D: Myocardial perfusion scans: projected population cancer risks from current levels of use in the United States. Circulation 2010, 122:2403 2410.

4. Medical Information Division IMV: 2008 Nuclear Medicine Market Summary Report. Des Plains, IL: IMV Medical Information Division; 2008

5. Anger HO: A New Instrument for Mapping Gamma-ray Emitters. Berkeley, CA: University of California Radiation Laboratory; 1957.

6. Herzog BA, Buechel RR, Katz R, Brueckner M, Husmann L, Burger IA, Pazhenkottil AP, Valenta I, Gaemperli O, Treyer V, Kaufmann PA: Nuclear myocardial perfusion imaging with a cadmium-zinc-telluride detector technique: optimized protocol for scan time reduction. J Nucl Med 2010, 51:46 51.

7. Ostrzega E, Maddahi J, Honma H, Crues JV 3rd, Resser KJ, Charuzi Y, Berman DS: Quantification of left ventricular myocardial mass in humans by nuclear magnetic resonance imaging. Am Heart J 1989, 2:444 452.

8. Imbert L, Poussier S, Franken PR, Songy B, Verger A, Morel O, Wolf D, Noel A, Karcher G, Marie PY: Compared performance of high-sensitivity cameras dedicated to myocardial perfusion SPECT: a comprehensive analysis of phantom and human images. J Nucl Med 2012, 53:1897 1903.

9. Gambhir SS, Berman DS, Ziffer J, Nagler M, Sandler M, Patton J, Hutton B, Sharir T, Haim SB, Haim SB: A novel high-sensitivity rapid-acquisition single-photon cardiac imaging camera. J Nucl Med 2009, 50:635 643

10. Bocher M, Blevis IM, Tsukerman L, Shrem Y, Kovalski G, Volokh L: A fast cardiac gamma camera with dynamic SPECT capabilities: design, system validation and future potential. Eur J Nucl Med Mol Imaging 2010, 37:1887 1902.

11. Higley B, Smith FW, Smith T, Gemmell HG, Das Gupta P, Gvozdanovic DV, Graham D, Hinge D, Davidson J, Lahir A: Technetium-99 m-1,2-bis[bis(2-ethoxyethyl) phosphino]ethane: human biodistribution, dosimetry and safety of a new myocardial perfusion imaging agent. J Nucl Med 1993, 34:30 38.

12. Dorbala S, Crugnale S, Yang D, Di Carli MF: Effect of body mass index on left ventricular cavity size and ejection fraction. Am J Cardiol 2006, 97:725 729.

13. Kennedy JA, Isreal O, Frenkel A: 3D iteratively reconstructed spatial resolution map and sensitivity characterization of a dedicated cardiac SPECT camera. J Nuclear Cardiol 2014, 21:443 452

14. Talley NJ, O'Connor S: Examination Medicine: A Guide to Physician Training. Edinburgh: Churchill Livingstone; 2009:41.

15. Metzler SD, Bowsher JE, Smith MF, Jaszczak RJ: Analytic determination of pinhole collimator sensitivity with penetration. IEEE Trans. Med. Imag 2001, 20:730 741.

16. Chahal NS, Lim TK, Jain P, Chambers JC, Kooner JS, Senior R: Population-based reference values for 3D echocardiographic LV volumes and ejection fraction. JACC Cardiovasc Imaging 2012, 5:1191 1197.

\section{Submit your manuscript to a SpringerOpen ${ }^{\circ}$ journal and bene区t from:}

- Convenient online submission

- Rigorous peer review

- Immediate publication on acceptance

- Open access: articles freely available online

- High visibility within the 囚eld

Retaining the copyright to your article 